BMJ Paediatrics Open

\title{
Paediatric clinical ethics in Australia and New Zealand: a survey
}

\author{
Emma Cottle, ${ }^{1}$ Melanie Jansen, ${ }^{2}$ Helen Irving, ${ }^{2}$ Ben Mathews ${ }^{3}$
}

To cite: Cottle E, Jansen M, Irving $\mathrm{H}$, et al. Paediatric clinical ethics in Australia and New Zealand: a survey. BMJ Paediatrics Open 2017:1:e000156. doi:10.1136/ bmjpo-2017-000156

- Prepublication history and additional material is available. To view please visit the journal (http://dx.doi.org/10.1136/ bmjpo-2017-000156).

Received 12 June 2017 Revised 18 July 2017 Accepted 19 July 2017

\section{(1) CrossMark}

${ }^{1}$ School of Medicine, Griffith University, Gold Coast, Queensland, Australia ${ }^{2}$ Centre for Children's Health Ethics and Law, Children's Health Queensland, Brisbane, Queensland, Australia ${ }^{3}$ Australian Centre for Health Law Research, Queensland University of Technology, Brisbane, Queensland, Australia

Correspondence to DrEmma Cottle; emma.cottle@ health.qld.gov.au

\section{ABSTRACTS}

Objectives To quantify the presence, purpose, function, governance and funding of clinical ethics services (CES) in tertiary paediatric hospitals in Australia and New Zealand. Design, setting and participants A descriptive, quantitative survey was conducted across eight paediatric hospitals.

Main outcome measures Responses from survey questions on the presence, purpose, function, governance and funding of the CES.

Results Seven of eight tertiary paediatric hospitals identified access to CES. Regarding purpose and function, all CES provided clinical case consultation, six of seven provided education and training, six of seven assisted with organisational policy and guideline development and four of seven undertook original ethics research. There was wide variation in how case consultations were conducted, reported and documented. With respect to governance and funding, all CES reported to their hospital executive and only one CES reported having a dedicated, although small, budget.

Conclusions Heterogeneity in the process of case consultation and CES policy content exists across the organisations studied. There is consistency with the broader values that underpin CES such as their multidisciplinary nature and level of training required for key staff. There is an apparent lack of formal budgetary support from health services for CES activities, with support derived mostly from staff who contribute their time in addition to their primary roles.

\section{INTRODUCTION}

Clinical ethics services (CES) have evolved to support ethical decision making in day-to-day clinical practice. They differ from Human Research Ethics Committees as they have no role in research protocol review. There are multiple models of CES provision, from formal committees that are convened to make a binding decision about a particular case, to those that purely provide retrospective case review for educational purposes. CES are considered an important resource by key healthcare bodies in Australia and internationally. ${ }^{1-4}$ In the USA, it is an accreditation requirement that hospitals have a mechanism to deal with clinical ethical issues. ${ }^{5}$ In the UK, there are no legislative requirements for hospitals to have CES, and only some European

\section{What is already known on this topic?}

Clinical ethics services (CES) are recommended in health service delivery guidelines in many developed countries.

- There is a paucity of data identifying and describing existing paediatric $\mathrm{CES}$.

- There is a need to develop service standards and benchmarks for CES.

\section{What this study hopes to add?}

There is wide variation in case consultation conduct, involvement of patients and families, and documentation of consultations among CES at tertiary children's hospitals.

- There are emerging points of consistency in practice: such as a multidisciplinary approach to consultation, and CES governance being independent of other clinical and administrative departments.

There is a lack of formal funding support from hospital health services.

countries, such as Belgium and Norway, mandate this. ${ }^{6}$ In Australia, there is little regulatory framework. The Australian Council on Healthcare Standards EQuIP National Guidelines recommends that a health service has a 'formal, nominated consultative entity where ethical decision making can be referred...' How this entity should operate and its specific role is not specified.

There is significant heterogeneity in the presence, purpose and function of CES both in Australia and internationally. ${ }^{7-15}$ CES may be provided by individual clinical ethicists (a title for which there is minimal regulation), or by a group response. Some CES function mainly for policy formation or education, whereas some focus primarily on case consultation. The literature describing paediatric CES is sparse. In Australia, there is one published study examining the CES at the Royal Children's Hospital, Melbourne. ${ }^{16}$ Internationally, one study examined the CES at the Zurich University Children's Hospital, ${ }^{17}$ 
and another study surveyed clinical ethicists at children's hospitals in the USA. ${ }^{18}$ They found that $60 \%$ of facilities had a policy for ethics consultation, although some 'elements of the national consensus statements were inconsistently included, 'and wide variation in salary, administrative and budget support exists. ${ }^{18}$

Worldwide, there are few specific standards to which CES must adhere. Valuable resources to support CES function are emerging, such as the UK Clinical Ethics Network Core Competencies for Clinical Ethics Committees ${ }^{4}$ and the National Health and Medical Research Council's Clinical Ethics Capacity Building Manual. ${ }^{19}$ These provide advice to organisations developing CES, but their recommendations are not binding. The lack of consistent standards for CES has led some to question their integrity. ${ }^{20}$ The development of consistent standards for CES operation would be a positive step to enhance their integrity. The authors agree with Godkin et al, that for clinical ethics to provide optimal integrated services, we need to gain knowledge of best practices and ways in which to measure effectiveness of the services. ${ }^{21}$

The first step in developing consistent CES standards is to identify current existence, purpose, function, governance and funding of CES. This study's objective is to provide a comprehensive description of these aspects of CES in tertiary paediatric hospitals in Australia and New Zealand. The results will aid in crafting future research to closely evaluate and further develop these services, to ensure that CES are a valuable resource, providing support to clinicians and contributing to improved patient outcomes.

\section{METHODS}

Informed by a literature review of CES in tertiary children's hospitals, a quantitative survey was developed (online supplementary file 1). Kesselheim and colleagues kindly shared their survey instrument with us, for use as a guidance document to develop our survey to suit the Australasian context. ${ }^{18}$ The online survey was tested by two staff members from the Centre for Children's Health Ethics and Law at Lady Cilento Children's Hospital for validity, readability and clarity.

Tertiary paediatric hospitals across Australia and New Zealand were considered eligible to participate. Separate neonatal units outside paediatric centres were excluded. The authors identified the most appropriate individuals at each facility to be invited to complete the survey, via discussion with staff at each hospital's CES or hospital executive. Participants were then invited to participate by email. Informed consent was gained at the beginning of the survey. Responses were collected between April and June 2016.

Nine tertiary paediatric hospitals were identified as eligible to participate in the study. Despite reasonable efforts, we were unable to engage one of the sites and so were unable to invite that site to participate.
Descriptive statistics (percentages) were automatically generated by the survey software from responses.

\section{RESULTS}

Eight children's hospitals participated. There was a $100 \%$ response rate from invited participants.

Seven of eight hospitals had access to a CES, and the majority of these were dedicated paediatric CES.

Six of seven CES provided general education and training such as grand rounds. One of seven CES provided education specific to nursing/allied health staff. Four of seven CES undertook original ethics research and six of seven were involved in policy and guideline development. All CES provided case consultation. The hospital without a formal CES handled ethical issues by seeking a second opinion from colleagues.

Key features of the case consultation process are included in table 1. Three of six CES used a group response and three of six CES used either a group response or an individual ethicist depending on the case type. The number of people in the group response pool who can respond to a referral varied. Of the six respondents, four had between 2 and 10 members in the pool, one had 11-15 members and one had more than 20 members. The representation required in the group response pools is shown in table 2 . The training required for the members varied from none to a $\mathrm{PhD} /$ Master's degree in bioethics (or similar). Two CES employed a clinical ethicist and required this person to have a $\mathrm{PhD} /$ Master's degree in bioethics, clinical ethics or moral philosophy, as well as on-the-job experience.

Referral processes across the centres studied were variable. Six of six CES accepted referrals from clinical staff and hospital administration/executive. Two of six accepted referrals from patients and two of six accepted referrals from parents or family members. No CES accepted referrals solely from the treating doctor.

Four of seven CES had a written policy on how to conduct a case consultation. There was significant heterogeneity in these, the content of which is summarised in table 3. In terms of the consultation model employed, two of six CES generated specific recommendations and two used a facilitative model. Two other CES used a combination of these options depending on the case type.

The response time following a referral varied. Three of six CES responded within 24hours, two CES responded within a 1-day to 1-week period and one CES had a response time of over 2 weeks.

Regarding the frequency of referrals, in the 12-month period preceding the survey, three of six CES had $11-15$ referrals, one had $6-10$ referrals and two had $1-5$ referrals.

Only one of the CES reported having a dedicated budget for their service. Time-limited funding was provided from a charity source and supported part funding for an administration officer and a part-time medical officer. All CES had clinical staff who were employed elsewhere in the health 
Table 2 Required representation on clinical ethics committee. Total respondents: 4

\begin{tabular}{ll}
\hline Answer choices & Responses \\
\hline Lawyer & 2 \\
\hline Allied healthcare professionals & 4 \\
\hline Nursing & 4 \\
Medical staff & 3 \\
\hline Philosopher & 1 \\
\hline Clinical ethicist & 3 \\
\hline Other university academics & 1 \\
Community member/layperson & 3 \\
\hline Chaplain/member of religious group & 3 \\
\hline
\end{tabular}

service but contributed time to the CES, and three of six CES had staff employed directly by the CES. Two of six CES had in-kind support from academics of partner universities, and two of six had support from hospital chaplains or representatives of religious communities.

All CES reported directly to the Health Service Executive/CEO or equivalent.

The lead person of all CES held a relevant $\mathrm{PhD} /$ Master's degree. In addition, most had completed an ethics minicourse. One lead person had been mentored by an experienced ethicist.

\section{DISCUSSION}

CES are available in most tertiary paediatric hospitals in this region. In the absence of CES, ethical issues are resolved by second opinion from other colleagues, which is broadly consistent with findings in other surveys of general hospitals in Australia. ${ }^{15}$
Gold et al describe the essential elements to CES functioning as follows: timely clinical case consultation, education and training, research and institution policy and guideline development. ${ }^{16}$ We found significant variation in these elements in Australian and New Zealand CES.

Most CES contributed to the education of general hospital staff through Grand Rounds, and many contributed formally to junior doctor education. Given that all the CES surveyed required that nursing and allied health staff be represented in their response groups, it is notable that only one CES provided education to these groups. Only just over half of the CES generated original ethics research. This may reflect the small number of staff directly employed by CES, with limited budgetary support making it difficult to devote adequate time to developing research ideas and applying for grants. Additionally,

Table 3 Content of written policy. Total respondents: 4

\begin{tabular}{ll}
\hline Answer choices & Responses \\
\hline $\begin{array}{l}\text { Who can request a consultation } \\
\text { How to contact the ethics service }\end{array}$ & 4 \\
$\begin{array}{l}\text { How the ethics service responds to requests } \\
\text { for consultation }\end{array}$ & 3 \\
$\begin{array}{l}\text { How the case meeting/deliberation is } \\
\text { conducted }\end{array}$ & 3 \\
$\begin{array}{l}\text { Who is included in the consultation } \\
\text { Methods for notification of affected persons }\end{array}$ & 3 \\
$\begin{array}{l}\text { Protection of patient confidentiality } \\
\text { How the consultation is documented }\end{array}$ & 1 \\
$\begin{array}{l}\text { Identification of patient groups/situations that } \\
\text { trigger an automatic consultation }\end{array}$ & 1 \\
How the service handles complaints & 0
\end{tabular}

Table 1 Key features of the case consultation process. Total respondents: 7

\begin{tabular}{|c|c|c|c|c|c|c|}
\hline & Never & Rarely & Sometimes & Usually & Always & Total \\
\hline Meet with more than one member of the clinical team & 0 & 0 & 0 & 1 & 6 & 7 \\
\hline Notify the patient and/or family about the consultation & 0 & 1 & 2 & 2 & 2 & 7 \\
\hline Meet with patients and/or families & 0 & 2 & 4 & 0 & 1 & 7 \\
\hline $\begin{array}{l}\text { Include the patient and/or family in the case meeting/ } \\
\text { discussion }\end{array}$ & 4 & 2 & 0 & 0 & 1 & 7 \\
\hline $\begin{array}{l}\text { Enter a written report of the consultation into the patient's } \\
\text { medical record }\end{array}$ & 4 & 0 & 0 & 0 & 3 & 7 \\
\hline Provide a written report of the consultation to the clinical team & 0 & 0 & 0 & 3 & 4 & 7 \\
\hline $\begin{array}{l}\text { Provide a written report of the consultation to the patient, } \\
\text { family or both }\end{array}$ & 1 & 1 & 2 & 2 & 1 & 7 \\
\hline $\begin{array}{l}\text { Follow-up with the participants after the consultation is } \\
\text { completed to receive clinical updates }\end{array}$ & 0 & 0 & 1 & 3 & 3 & 7 \\
\hline $\begin{array}{l}\text { Follow-up with the participants after the consultation is } \\
\text { completed to receive feedback on the consultation }\end{array}$ & 0 & 1 & 0 & 1 & 5 & 7 \\
\hline $\begin{array}{l}\text { Participate in formal review of clinical ethics consultations for } \\
\text { educational and/or quality assessment purposes }\end{array}$ & 2 & 2 & 0 & 2 & 1 & 7 \\
\hline
\end{tabular}


clinical ethics is a fledgling field in this region, with relatively fewer people with the required expertise to drive projects in this space.

Case consultation was a ubiquitous activity of all the CES surveyed. This is in contrast to findings in McNeill's 2001 study where few Australian clinical ethics committees provided case consultation. ${ }^{15}$ This may reflect general developments in practice in the period since that study, or greater activity in case consultation within paediatric hospitals. Multidisciplinary group responses were the predominant approach to consultation, however apart from this there was little consistency in processes. Of note, three of the seven CES did not have a written policy for how to conduct a consultation. This is similar to Kesselheim et al's study which identified that $40 \%$ of US paediatric facilities lacked a policy for ethics consultation. ${ }^{18}$ There was also variation in the level of involvement of patients and family members. Two CES always notified the patient/family that the consultation was occurring, one CES always met with the patient/family, two CES accepted referrals from patients, and two CES accepted referrals from family members. While all surveyed CES required members to have on-the-job experience, there was no consistency in requirements for formal training. Despite this, there was a high standard of formal qualifications among those leading a CES, with all respondents having at least a relevant Master's degree level qualification. With regard to review processes, only three CES usually or always held formal review of CES consultations for education and quality improvement purposes.

This heterogeneity of practice is not unique to Australian CES. Myers and Lantos noted inconsistency in their US-based study, and suggested that the lack of consistent standards for CES raises significant concerns about the integrity of ethics consultation. ${ }^{20}$ We hold that heterogeneity in approach to consultation is not necessarily negative, as CES need to be responsive to local needs and appropriate to local culture. For example, the best consultation approach may differ in a community aged-care setting, compared with that best suited for an obstetric unit in a regional hospital. Variation in clinical ethics practice is both inevitable and appropriate. However, this should be balanced with the need for an important healthcare service to function (and to be perceived to function) with rigour and transparency-so that healthcare peers and the broader community can have clear expectations of CES and trust their integrity. One way that an accreditation body could promote this goal is to require CES to have formal terms of reference which detail their processes (and rationale for these processes) without necessarily imposing a particular consultation model. This approach would allow appropriate tailoring of CES to their local setting, but provides all stakeholders with transparency and a model against which to provide feedback.

While variation in consultative processes can be positive, our study discovered aspects of practice that we believe need both legal clarification and practice standardisation. A key concerning finding is the variability in documentation, with four of seven CES never documenting consultations in the medical record. This figure was $12 \%$ in Kesselheim et als study. ${ }^{18}$ If CES are considered a legitimate clinical service, it is problematic if consultations are not routinely documented, particularly given the case complexities that often underlie the request for a CES consultation. Thorough and timely documentation is good practice from both a medicolegal and clinical perspective and increased consistency in this should be an aim across the clinical ethics community.

Only one CES reported having its own budget. However, the survey wording of 'own budget' may have been subject to different interpretation. Given that three CES reported having staff employed by the CES, a budget clearly existed. Nevertheless, the only funding available for these CES is reportedly through charity organisations rather than from hospital funding obtained from the state health budget. This is a salient finding, given the importance placed on CES by accreditation bodies. ${ }^{1}$ In contrast, Kesselheim et $a$ 's study reported $24 \%$ of CES had a dedicated budget and $33 \%$ had salary support. ${ }^{18}$ Overwhelmingly, those who contribute to the functioning of CES do so in addition to their usual clinical/ pastoral role in the hospital, or their academic role at universities. We think this is positive, as it means CES are very engaged with frontline health practitioners and university partners, and clinical and academic staff have rich opportunities to gain clinical ethics consultation experience. However, perhaps the balance needs to be adjusted so that more core clinical ethics staff are paid by the CES, so that these services are not so dependent on in-kind support from time-challenged clinicians and academics. Having dedicated staff may also mean that CES have greater capacity to run education programme $\mathrm{s}$ and pursue research agendas, thus contributing to the ongoing development of the field.

Governance of CES was uniform, with all services reporting directly to the hospital's executive. Although the academic qualifications of the CES lead staff member were generally high, the level of required clinical experience varied, with only one person having been mentored by an experienced ethicist. This figure was lower than the $36 \%$ reported in Kesselheim et al's study, ${ }^{18}$ and may reflect the lack of experienced clinical ethicists in the region. Overall, CES appear to be separate from other departments. We think this is positive, as it is likely to enable better cross-specialty consultation and situates clinical ethics as a service that has overarching impact on healthcare provision across the health service.

\section{Limitations of the study}

The study has two limitations. First, despite all reasonable efforts, one eligible service was not able to be invited to participate. Nevertheless, all other tertiary paediatric hospitals in the region participated, hence the results are considered to be broadly representative of the region. 


\section{Box Recommendations}

1. To promote transparency, clinical ethics services (CES) should have terms of reference that detail their approach to consultation and the rationale for this.

2. To ensure clinical and medicolegal best practice, documentation of clinical ethics consultations should be clarified and standardised.

3. Identification of recurrent funding sources will enable sustainable services that have increased scope for providing education and pursuing research.

4. Further research into methods of case consultation, education practices and efficacy of CES is required.

Second, responses to questions about the CES budget indicated participants had different interpretations of this question. This has resulted in difficulty in interpreting this part of the data.

\section{Future research directions}

Observational studies similar to this in the general hospital, rural/remote and community settings would give a broader picture of CES and contribute to the development of national guidelines. Now that we have a snapshot of CES in the paediatric setting, analysis of different approaches to case consultation and education, including ways to measure efficacy, is essential in developing an evidence base for CES provision (box).

Contributors EC and MJ were responsible for a literature review, drafting and editing of the research protocol, drafting and editing of the survey instrument, recruiting participating facilities, and drafting and editing of the final article. HI and BM were responsible for editing the research protocol, the survey instrument and the final article. This study was undertaken by a final year MD student, supervised and coauthored by members of CCHEL in compliance with the ICMJE Recommendations. All authors had access to all of the data.

Funding Funding of this study fell under the remit of CCHEL. All authors had full access to all of the data.

Competing interests None declared.

Ethics approval Ethics approval was granted by the following HRECs: Sydney Children's Hospitals Network (LNR/15/SCHN/428, 18/11/2015), Women's and Children's Hospital Network (HREC/15/WCHN/194, 11/03/2016), Queensland University of Technology (1500000971, 13/11/2015), Children's Health Queensland (HREC/15/QRCH/171, 15/09/2015). Formal HREC approval was not required by Monash Health, Royal Children's Hospital or Princess Margaret Hospital for Children.

Provenance and peer review Not commissioned; externally peer reviewed.

Data sharing statement There are no additional unpublished data from this study.

Open Access This is an Open Access article distributed in accordance with the Creative Commons Attribution Non Commercial (CC BY-NC 4.0) license, which permits others to distribute, remix, adapt, build upon this work non-commercially, and license their derivative works on different terms, provided the original work is properly cited and the use is non-commercial. See: http://creativecommons.org/ licenses/by-nc/4.0/ (c) Article author(s) (or their employer(s) unless otherwise stated in the text of the article) 2017. All rights reserved. No commercial use is permitted unless otherwise expressly granted.

\section{REFERENCES}

1. The Australian Council on Healthcare Standards (ACHS). EQuIPNational guidelines standard 15. Sydney Australia: ACHS, 2012.

2. Royal College of Physicians of London. Ethics in practice: background and recommendations for enhanced support. Wiltshire: Royal College of Physicians, 2005.

3. Australian Health Ethics Committee. AHEC consensus statement on clinical ethics. Canberra (AU): National Health and Medical Research Council, 2015:1. https://www.nhmrc.gov.au/health-ethics/ethicalissues-and-further-resources/ahec-consensus-statement-clinicalethics. (accessed 22 Apr 2016).

4. UK Clinical Ethics Network. Core competencies for clinical ethics committees: a consensus statement from the UK clinical ethics network. 2007:10 http://www.ukcen.net/uploads/docs/education resources/core_competencies1.pdf (accessed 22 Apr 2016).

5. Joint Commission on Accreditation of Healthcare Organizations. Accreditation manual for hospitals. Oakbrook Terrace, IL: Joint Commission on Accreditation of Healthcare Organizations, 1993. Cited by Slowther A, Hope T. Clinical ethics committees: they can change clinical practice but need evaluation. BMJ 2000;312:649-650.

6. UK Clinical Ethics Network. Practical guide to clinical ethics support. European experience section. http://www.ukcen.net/education_ resources/support_guide/section_a_clinical_ethics_support. (accessed 2015 Apr 20).

7. Berchelmann K, Blechner B. Searching for effectiveness: the functioning of connecticut clinical ethics committees. J Clin Ethics 2002;13:131-45.

8. Hoffmann DE. Evaluating ethics committees: a view from the outside. Milbank Q 1993;71:677-701.

9. Orr RD, Moon E. Effectiveness of an ethics consultation service. $J$ Fam Pract 1993;36:49-53.

10. Slowther A, Bunch C, Woolnough B, et al. Clinical ethics support services in the UK: an investigation of the current provision of ethics support to health professionals in the UK. J Med Ethics 2001;27(Suppl 1):2i-8.

11. Storch JL, Griener GG. Ethics committees in Canadian hospitals: report of the 1990 pilot study. Healthc Manage Forum 1992;5:19-26.

12. van der Kloot Meijburg HH, ter Meulen $\mathrm{RH}$. Developing standards for institutional ethics committees: lessons from The Netherlands. J Med Ethics 2001;27(Suppl 1):36i-40.

13. White BD, Zaner RM, Bliton MJ, et al. An account of the usefulness of a pilot clinical ethics program at a community hospital. QRB Qual Rev Bull 1993;19:17-24.

14. Liu J, Shen J, Liu PZ. A survey of five first-level hospital ethics committees in Urumqi, China. J Zhejiang Univ Sci B 2013;14:541-8.

15. McNeill PM. A critical analysis of Australian clinical ethics committees and the functions they serve. Bioethics 2001;15:443-60.

16. Gold H, Hall G, Gillam L. Role and function of a paediatric clinical ethics service: experiences at the Royal Children's Hospital, Melbourne. J Paediatr Child Health 2011;47:632-6.

17. Streuli JC, Staubli G, Pfändler-Poletti M, et al. Five-year experience of clinical ethics consultations in a pediatric teaching hospital. Eur J Pediatr 2014;173:629-36.

18. Kesselheim JC, Johnson J, Joffe S. Ethics consultation in children's hospitals: results from a survey of pediatric clinical ethicists. Pediatrics 2010;125:742-6.

19. Australian Health Ethics Committee. Clinical ethics capacity building resource manual. Canberra (AU: National Health and Medical Research Council, 2015:44. https://www.nhmrc.gov.au/guidelinespublications/e114 (accessed 18 Oct 2016).

20. Myers D, Lantos J. The slow, steady development of pediatric ethics committees, 1975-2013. Pediatr Rev 2014;35:e15-e9.

21. Godkin MD, Faith K, Upshur RE, et al. Project examining effectiveness in clinical ethics (PEECE): phase 1- descriptive analysis of nine clinical ethics services. J Med Ethics 2005;31:505-12. 